\title{
DIRECTIONAL ANTENNA BASED PERFORMANCE EVALUATION OF 802.11 WIRELESS LOCAL AREA NETWORKS
}

\author{
Kartikeya Tripathi, Janise McNair and Haniph Latchman \\ Department of Electrical and Computer Engineering,University of Florida, \\ Gainesville, Florida 32611
}

\begin{abstract}
In this paper, the use of directional antennae on access points in wireless local area networks to sectorize the coverage area is proposed. This is shown to be a simple way to significantly increase throughput while greatly reducing the occurrence of the hidden terminal problem. First, we discuss the current use of sectorization in various wireless networks, including cellular and ad hoc networks. Then, we present the details of the proposed architecture, describing the necessary enhancements to the current Access Point functionality and examining the tradeoffs of costs and infrastructure. Finally, we carry out a performance analysis to demonstrate the marked increase in throughput and efficiency achieved by the new technique.
\end{abstract}

Key words: Wireless Local Area Network (WLAN); 802.11; sectorization; Access Point (AP); Performance Analysis

\section{INTRODUCTION}

Over the last five years, wireless local area networks (WLANs) have become an increasingly popular choice for users seeking data services both at home and at the office. The reason for the success is that WLANs can support highly crowded, hot spot areas needing high data rates with a minimal investment in infrastructure, functioning as a wireless extension of the Ethernet. However, WLANs still suffer from reduced data rates due to unreliable wireless links, error control overhead, and overhead for medium 
access control. Though standards such as $802.11 \mathrm{a} / \mathrm{g}$ are evolving to achieve higher data rates by using orthogonal frequency division multiplexing (OFDM) at the physical layer, the 802.11 MAC continues to rely on an omni-directional access point (AP) that runs one instance of the carrier sense multiple access with collision avoidance (CSMA/CA) protocol. This approach has become a bottleneck for high-speed data and multimedia applications because of the increased overhead required to coordinate user access. In fact, the overhead incurred by RTS and CTS decreases the actual throughput of 802.11 to almost half the throughput that can be achieved at the physical layer.

A common technique used to overcome this problem, i.e., to increase WLAN throughput in high traffic areas, is to use several co-located APs to serve one network in an extended service set (ESS). For example, since the $802.11 \mathrm{~b}$ standard specifies three non-overlapping frequency channels, three APs can be co-located, each using a different channel, allowing three simultaneous instances of 802.11 , and improving the overall throughput. Similar results can be seen with the 802.1 la standard, which can provide up to 8 orthogonal channels. In fact, multiple co-located APs for 802.11a are necessitated due to the smaller coverage area of the same. Though this procedure can solve the problem to some extent, requiring multiple APs to serve one location is an inefficient use of hardware, and points to an inherent capacity ceiling. However, with small improvements in the software and the hardware, a single AP can use the idea of a simple router to significantly multiply the achievable bandwidth at the last hop wireless link.

In this paper, we propose a new sectorized architecture for infrastructurebased WLANs. In Section 2, we discuss the current use of sectorization in various wireless networks, including cellular and ad hoc networks. Then, in Section 3, we describe the proposed modifications, including necessary enhancements to current AP functionality, and an examination of the tradeoffs with respect to costs and infrastructure. Section 4 provides a description of the performance analysis used to determine the effectiveness of the new scheme, followed by the numerical results in Section 5. Finally, Section 6 provides some conclusions and a discussion of future work.

\section{RELATED WORK}

A detailed description of the 802.11 medium access control (MAC) protocol can be found in [1], [2]. Quality of Servive (QoS) guarantees for delay sensitive traffic and the enhancement of the throughput in the 802.11 protocol have been topics of intensive research. Most of the new ideas in this area, as in [3], try to suggest some improvement in the system performance 
by making variations in the traditional CSMA/CA scheme for medium access control. Similarly, many mobility models and measures have been proposed for simulating moving terminals in a generic wireless coverage area, as in [4]. The use of directional antennas for WLAN access points has been studied in [5] and [9] as a space division multiple accesses (SDMA) technique to increase the capacity of cellular and WLAN communication systems respectively. Cell sectorization using directional antennas has often been implemented in cellular voice networks to increase the frequency reuse factor at the cost of a decrease in trunking efficiency [6]. In [7], a new MAC protocol for ad hoc wireless LANs was proposed, which was based on the CSMA/CA scheme using directional antennas. This scheme uses the idea of directional RTS and CTS to allow simultaneous conversations, which do not interfere with each other, and hence improve the throughput. However, little work has been done on medium access control for sectorized access points in infrastructure-based wireless LANs.

\section{SECTORIZING THE COVERAGE AREA}

\subsection{Enhanced AP Functionality}

The AP in the current 802.11 topology is a layer- 2 device, which has only two network ports: one to connect it to the wired Ethernet, and the other to connect it to the radio interface. Such a design is effective for a small office environment, or for home networking, both of which serve a small number of mobile units (MUs). However, as 802.11 becomes a data networking solution for larger organizations with a large number of MUs, and also the possible $4 \mathrm{G}$ wireless solution, the simple AP design loses its efficiency. We propose the design of 802.11 APs with one Ethernet port and multiple radio ports, where each radio interface has a semi-directional antenna, covering, say, 360 degrees in the vertical and 90 degrees in the horizontal. Each directional antenna can be configured on one of the orthogonal channels ( 3 in 802.11 b) to allow the data in each sector to be transmitted and received separately on each antenna. The antennas can be placed in a spatially predetermined way to minimize co-channel interference. Figure 1 shows the sectorized AP configuration for four directional antennas. 


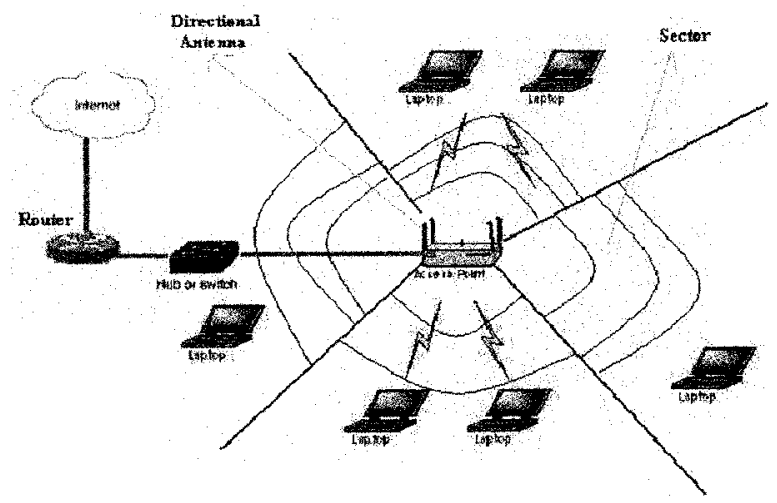

Figure 1. Sectorized AP Configuration for Infrastructured WLANs

\subsection{Medium Access Control}

We envision a separate MAC instance running in each of the sectors at the AP. The MUs in each sector contend amongst each other using CSMA/CA to gain access to their respective directional antennas. We do not expect buffering to be a major issue. For example, to run three instances of $802.11 \mathrm{~b}$ at the AP, the Ethernet port will support $100 \mathrm{Mbps}$ links, while each directional antenna can have a maximum rate of $11 \mathrm{Mbps}$. We propose that the sectorized AP use a sector table, similar to a routing table, to route the downlink traffic efficiently. This table will comprise the current sector and the MAC address for each MU. As MUs move around the coverage area, this table will have to be updated every certain interval of time.

\subsection{Trade-offs}

In the implementation of the sectorized scheme, we expect to observe the following advantages:

- Since each single sectorized AP has multiple conversations taking place at the same time, the throughput can be increased.

- The hidden node problem will be reduced by sectorization, since there is a much smaller probability that MUs in the same sector cannot hear each other.

- An added benefit to the reduction of the hidden terminal problem is that the need for RTS/CTS overhead is eliminated, again increasing the throughput performance. 
On the other hand, there are several issues to be considered for implementing sectorized APs, including:

- The new approach would significantly increase the computational complexity of the AP, which was originally built to be a simple device. However, considering the increasing demand for high bandwidth wireless services, the application of WLANs to high traffic areas, and the objective to provide QoS for multimedia traffic, the customer satisfaction resulting from increased throughput should offset the investment in added complexity.

- Similar to the above issue, the design of a new sectorized AP would be more costly for manufacturers and customers alike. However, considering the new technique as a replacement for the practice of using several APs in a single location, the reduced number of APs should offset the additional hardware cost. In addition, mass manufacturing of electronic components tends to reduce the cost in the long run.

- Having sectors would bring forth the issues of user mobility and its influences on the effectiveness on the system. In particular, as MUs move among various sectors, each cross over results in control signaling and updating of the AP's sector table, which takes away time from useful data processing. There are also fluctuations in the throughput of the system as the load in each sector varies, and this depends on the patterns of mobility of the users.

- Finally, if many MUs gather in a concentrated area, such as an audience in an auditorium or conference room, then there would be no activity in several sectors, while one or two sectors may be overloaded. Some cases are shown in figure 2 . In this case, since the antennas are configured on different frequencies, each can be temporarily turned toward the problem sector to provide relief. Furthermore, a controlled feedback system can be designed to automatically change the orientation of the antennas, based on sector load and user density.

In light of the tradeoffs, and to quantify both the advantages and some of the pressing issues, we now evaluate the performance of the new technique. 

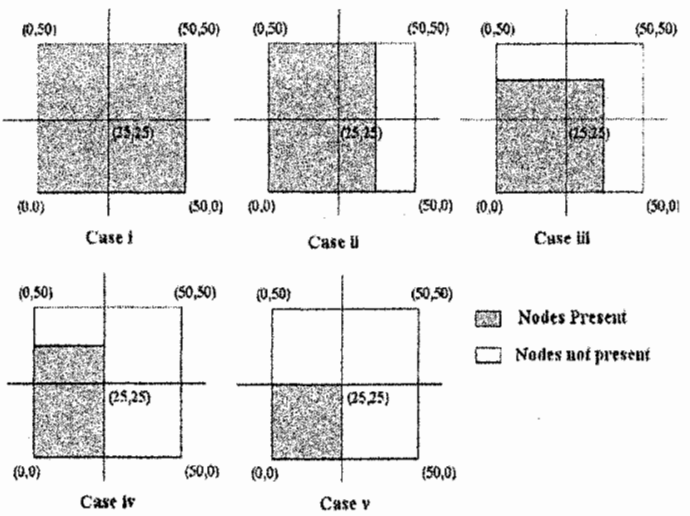

Figure 2. Variation in Node Density in a coverage area of $50 \times 50$ units

\section{PERFORMANCE ANALYSIS}

A discrete event analysis is used to analyze the performance of the scheme. We simulate the access behavior of $N$ stations located in an $L x L$ square unit area, with an $S$-sector AP located in the center.

\subsection{Fading Model}

The channel fading model used in test simulations is based on lognormal fading [8]. Log-normal model is a generic slow fading model. For this model, the path loss, $L_{P}$, in the signal propagation from node $\mathbf{i}$ to node $\mathbf{j}$ (and from AP to node) is given by:

$$
L_{P, i, j}=L_{O}+10 \alpha \log d_{i, j}+X
$$

where $L_{0}$ is the path loss at the distance of 1 meter, $\alpha$ is the path loss exponent, $d_{i, j}$ is the distance between nodes $\mathrm{i}$ and $\mathrm{j}$, and $X$ is a random variable based on a log-normal fading distribution.

\subsection{Hidden Node Calculation}

A signal transmitted from one MU can be missed by another MU if it is so far away from the transmitter that the signal fades till it reaches the other MU. This can lead to collisions when the transmissions from 2 MUs hidden 
from each overlap. The signal fading is on the basis of the model described above. If the received power from node $i$ to node $j$ is less than a pre-set threshold, then nodes $i$ and $j$ are considered to be hidden from each other.

\subsection{Collision Avoidance Calculations}

The CSMA/CA protocol is used independently in each sector of the AP, along with the corresponding 802.11 back-off procedure [2]. Every node is assigned a contention window, based on a uniform distribution from a lower value of 0 to an upper value of $\mathrm{CW}_{\min }-1$ time slots. Each time a collision occurs, the contention window is doubled, up to a maximum of $\mathrm{CW}_{\max ^{-1}}$ time slots. A record is kept of the number of successful transmissions and number of collisions.

\subsection{Throughput}

The net throughput of the system is calculated in terms of the percentage of total simulation time used for successful frame transmissions:

$$
\text { Throughput }=\frac{s \times t_{\text {FRAME }}}{T_{S I M}}
$$

where $s$ is the number of successful transmissions, $t_{F R A M E}$ is the transmission time of each frame, and $T_{S I M}$ is the total simulation time.

\section{RESULTS}

\subsection{Throughput and Hidden Nodes}

Numerical results for the throughput and for the number of hidden nodes are obtained for the sectored coverage area, and are compared with those for the traditional 802.11 omni-directional AP. This set of results is obtained for fixed MUs, all of which are distributed uniformly in the composite coverage area (except in cases with different distribution densities). Table 1 shows the chosen parameters used in the simulation. The significance of the node density is to capture the impact of user positioning on the performance of the system.

Figure 3 shows the throughput results comparing the use of a three-sector AP with the omni-directional AP, for a varying number of nodes. The three- 
sector case has a rapid increase initially, due to the smaller number of nodes being distributed among different sectors, thereby reducing the number of collisions. As the number of nodes increases, the number of collisions also increases, resulting in a gradual throughput reduction for the three-sector case. Yet, even with the decrease, the three-sector throughput has a significant improvement upon the performance of the omni-directional case.

Table 1. Simulation Parameters

\begin{tabular}{ll}
\hline Parameter Description & Value \\
Path loss factor $(\alpha)$ & 3 \\
Square area $(\mathrm{LxL})$ & $\mathrm{L}=50$ meters \\
Node Densities & Cases $1-5$ (see Figure 2) \\
Slot time & 50 micro seconds \\
DIFS & 128 micro seconds \\
Packet Size & 1 KB (constant) \\
\hline
\end{tabular}

Figure 4 shows the number of hidden terminal incidents for the sectored APs compared to the omni-directional case for the log normal fading model. It demonstrates that the number of hidden nodes is greatly reduced with an increase in the number of sectors used. As the coverage area per sector decreases, the MUs in a sector are closer and are less prone to being hidden from each other.

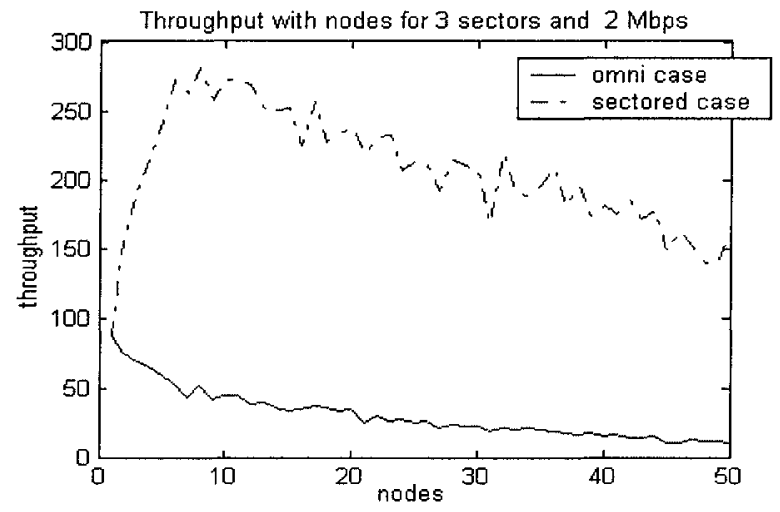

Figure 3. Throughput versus Total Number of Nodes (Density Case i) 


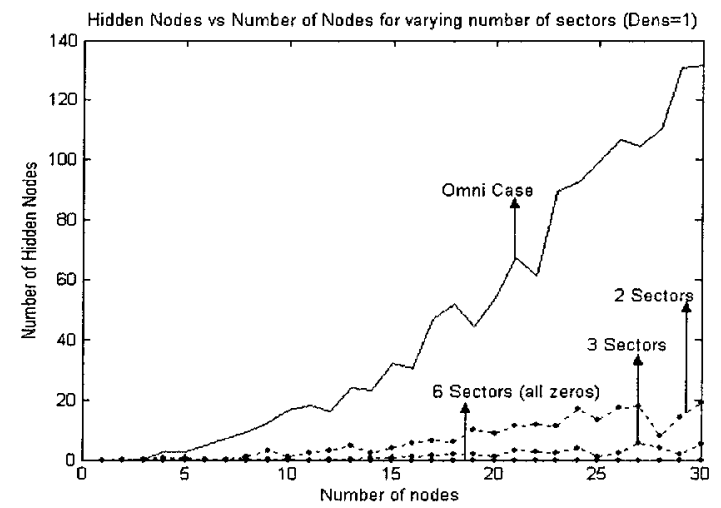

Figure 4. Number of hidden nodes vs. total number of nodes for Log-Normal Fading

The results in figures 3 and 4 were calculated for node density case i. As mentioned previously, one of the tradeoffs with the architecture is the effect of the node density on the performance of each sector. Figure 5 compares the throughput versus the number of sectors for various node densities, according to the cases illustrated in figure 2. It can be seen that the node density has minimal overall effect on the omni-directional throughput, while it has a drastic effect on the sectored AP throughput. The more concentrated the users are geographically, the more drastic the reduction.

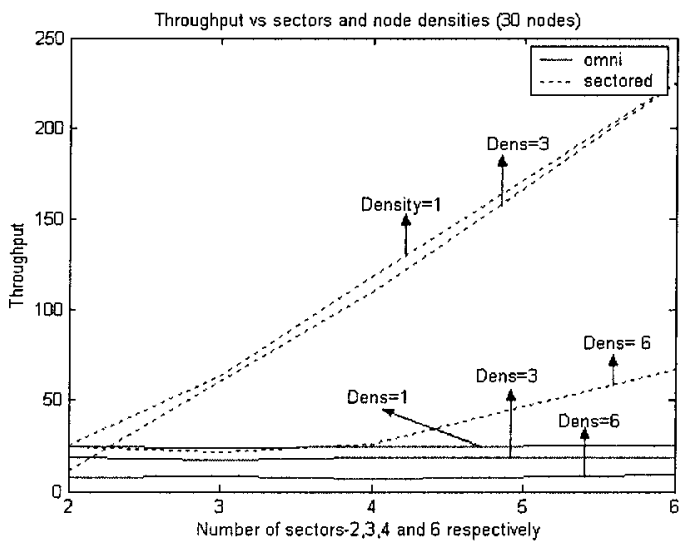

Figure 5. Comparison of Throughput vs. number of sectors for Log-Normal Fading 


\section{CONCLUSIONS}

As the demand for wireless bandwidth increases with each new hot application or network, the efficient use of network resources, including hardware, becomes more and more important. In this paper, it was demonstrated that the use of sectorized APs in WLANs is an effective way to increase throughput as well as to prevent incidences of hidden terminals. The scheme coordinates multiple instances of CSMA/CA running on a sectored AP, and provides a sector table for simple AP routing functions. The enhanced functionality for the AP introduces several tradeoffs, such as increased complexity and cost, that we believe are far out-weighed by the dramatic increases in throughput and efficiency, translating into a system that can handle an increased customer base with higher customer satisfaction.

\section{REFERENCES}

1. B.P. Crow, I. Widjaja, L.G. Kim, P.T. Sakai, "IEEE 802.11: Wireless Local Area Networks," IEEE communications Magazine, vol. 35, no. 9, pp. $116-126$, September 1997.

2. "Wireless LAN medium access control (MAC) and physical layer (PHY) specifications," Draft Standard, IEEE 802.11, P802.11/D1, 1997.

3. J.-L.C.Wu, H.-H. Liu, and Y.-J. Lung, "An adaptive multirate IEEE 802.11 wireless LAN," in Proc of International Conference on Information Networking, pp. 411-418, 2001 .

4. Eun-Seon Cho, Go-Whan Jin, Cheol-Hye Cho, "Comparisons of mobility models in cellular systems," Vehicular Technology Conference, pp. 19-22, September 1999.

5. F. Shad, T. Todd, V. Kezys, and J. Litva, "Indoor SDMA Capacity Using a Smart Antenna Basestation," in Proc, of IEEE ICUPC.97, pp. 868-872, 1997.

6. T.S. Rappaport, Wireless Communications: Principles and Practice, $2^{\text {nd }}$ edition, Prentice Hall, Upper Saddle River, New Jersey, 2002.

7. Y.-B. Ko, V. Shankarkumar and N.H. Vaidya, "Medium Access Control Protocols Using Directional Antennas in Ad Hoc Networks", IEEE INFOCOM, pp. 13-21, March, 2000.

8. K. Pahlavan, P. Krishnamurthy, Principles of Wireless Networks, 1st edition, Pearson Education, Singapore, 2002.

9. Bandyopadhyay, S.; Pal, M.N.; Saha, D.; Ueda, T.; Hasuike, K.; Pal, R, "Improving system performance of ad hoc wireless network with directional antenna", IEEE International Conference on Communications, Volume 2, May 2003. 